\title{
A coating-free superhydrophobic sensing material for full-range human motion and microliter droplet impact detection
}

Shanshan Jia ${ }^{a, b}$, Songlin Deng ${ }^{a}$, Yan Qing ${ }^{a, c,}{ }^{*}$, Guanjie He $^{b}$, Xunhe Deng ${ }^{a}$, Sha Luo ${ }^{a}$, Yiqiang Wu ${ }^{a, c, *}$, Jian Guo ${ }^{b}$, Claire J. Carmalt ${ }^{b}$, Yao Lu ${ }^{d, *}$ and Ivan P. Parkin ${ }^{b, *}$

${ }^{a}$ College of Materials Science and Engineering, Central South University of Forestry and Technology, Changsha 410004, P. R. China

b Department of Chemistry, University College London, 20 Gordon Street, London, WC1H 0AJ, UK

${ }^{\mathrm{c}}$ Hunan Provincial Collaborative Innovation Center for High-efficiency Utilization of Wood and Bamboo Resources, Central South University of Forestry and Technology, Changsha 410004, P. R. China

d Department of Chemistry, School of Biological and Chemical Sciences, Queen Mary University of London, Mile End Road, London, E1 4NS, UK

*Corresponding Authors

Email address: qingyan0429@163.com, wuyq0506@126.com, yao.lu@qmul.ac.uk, i.p.parkin@ucl.ac.uk 


\section{Abstract}

Traditional waterproofing strategies (e.g. plastic seals, superhydrophobic coatings) of strain sensors greatly limit their sensing performance (e.g., sensitivity, working-range, and working-life). Here a unique ultra-stretchable, coating-free superhydrophobic material is developed for high-performence strain sensing in harsh environments. This material integrates high sensitivity (GF of 2.1 to 214 ), wide sensing range (up to $447 \%$ strain), low resolution ( $<0.2 \%$ strain), dynamic durability (over 10000 stretching cycles at 50\% strain), and ultra-robust superhydrophobicity (mechanically, chemically, thermally, and UV impervious) in a single system, outperforming most of reported waterproof sensors. Such remarkable sensing materials can detect full range human movement, pulse rate and vocal fold vibration. The sensing material is designed to be superhydrophobic throughout its bulk material for work in harsh environments (water, corrosive liquid, high humidity, etc.). More importantly, the superhydrophobicity enables the highly sensitive sensor to detect microliter droplets impact with minimized energy loss. Thus, this sensing material should find many potential applications in wearable electronics, measurement platform, rainfall monitoring and intelligent irrigation system.

KEYWORDS: superhydrophobic, robustness, wearable sensor, high sensing performance, harsh environment applications, microliter droplets sensing 


\section{Introduction}

Electronic devices with flexible and soft features are a growing area for nextgeneration electronic devices. $^{[1,2,3,4]}$ In particular, stretchable, wearable and skinmountable strain sensors are needed for multiple applications as health monitors, ${ }^{[5]}$ electronic skin, ${ }^{[6]}$ artificial muscle, ${ }^{[7]}$ soft robotics ${ }^{[8,9]}$ etc. Sensing performance parameters, especially high sensitivity, broad sensing range, excellent reliability and durability, are crucial for practical utilization of these products since miniscule/large, frequent and long-duration strains are often applied to the sensor during applications. Additionally, these sensors are often required to operate under harsh environments, such as UV irradiation, sweat, corrosive liquids, humidity, high temperatures and water immersion. Flexible and wearable strain sensors are generally composed of conductive sensing elements (e.g., carbon materials, metal-organic frameworks) coupled with stretchable substrate materials (e.g., silicone rubber, fiber). ${ }^{[10,11,12,13,14,15,16,17]}$ However, harsh environments can lead to material degradation, fatigue and failure. ${ }^{[18,19]}$

To protect sensing materials from harsh environments, state-of-the-art strain sensors (commercial such as Electrovac co. 1td or laboratory-based) usually apply plastic seals/coatings. ${ }^{[20,21]}$ Although most plastic seals/coatings are effective to protect sensors from water, it is challenging to overcome harsh environments. Moreover, such plastic seals/coatings easily impair sensing performance, e.g. sensitivity and detection 
limit since the coating layer physically restricts the responding space of the sensing component. ${ }^{[22]}$

Superhydrophobic coatings are used as an alternative to plastic waterproofing for strain sensing materials to protect the sensors from harsh environments. ${ }^{[19,22,23,24,25,26,27,28,29,30,31]}$ These achievements are indeed impressive, however, strain sensors with simultaneous demonstration of all the aforementioned desirable sensing performance and excellent mechanical performance remains a challenge. This is mostly because they are made of multiple layers; superhydrophobic structure is fragile and sensing networks are easily removed from elastic substrates under large, repeated or long term dynamic strains. ${ }^{[32,33]}$

Here we show with an effective sensing material construction strategy that a durable coating-free superhydrophobic sensing material was achieved by integrating conductive layer, superhydrophobic layer, stretchable polymer into a single system. The superhydrophobic sensing material has high sensitivity, low detection limit, robust dynamic durability, wide sensing range, and is mechanically, chemically, thermally, and UV durable, through homogeneously encapsulating multi-walled carbon nanotubes (MWCNTs) in room temperature vulcanized silicone rubber (RTV), followed by a roughening process to create a superhydrophobic surface. The durable superhydrophobic surfaces enable the MWCNT/RTV sensing (MRS) material to work in a wide range harsh environments. More importantly, superhydrophobic surfaces designed here is for helping the sensor response water impacts with minimized energy 
dissipation via water bouncing on its surface, which has great potential to broaden the application scope for the sensor. This multifunctional MRS material applied as a wearable sensor to monitor a wide-range of human activities such as wrist, finger and knuckle movements, and physiological responses including pulse rate and speech, has been demonstrated.

\section{Results and discussion}

\subsection{Preparation and Characterization of the coating-free MRS materials}

The MRS material was prepared as illustrated in Figure 1a. First, MWCNTs were dispersed in cyclohexane with the assistance of strong sonication, followed by dissolving RTV and its curing agent through magnetic stirring and sonication to prepare a suspension. Then, the suspension was cast in a hand-folded paper box and cured at ambient condition to obtain the ultra-stretchable MWCNT/RTV composite. The MWCNTs were selected for their unique ability to form excellent percolation networks, even at high strain, by virtue of their high intrinsic inter-tube electrical conductivity, high aspect ratios and excellent mechanical properties. ${ }^{[18,32]} \mathrm{RTV}$ has great flexibility over a wide range of temperature, strong adhesion, and an intrinsic hydrophobicity, with reversible elastic deformation under harsh conditions and tuning of the surface energy, enhancing the anchoring strength of the MWCNT networks. ${ }^{[34]}$ A paper box was used as a mold to improve the primary roughness of the composites. Scanning electron microscope (SEM) images show that the bottom surface of the composites was rough due to marks transferred from the paper (Figure 1b). Without 
the printing process, the top surface was smooth (Supplementary Figure S1a), with good agreement with the results of atomic force microscope (AFM) test (Figure 2, details seen Supplementary Note 1). After being rubbed with sandpaper, both the top and bottom achieved a Lotus leaf-like dual-scale roughness created by continuous large protuberances with surface densely packed small features (Figure 1c-d and Supplementary Figure S1b). However, texturing the bottom surface was much faster, less than $1 / 5$ of the times than time spent on the top surface (Supplementary Movie S1). The hierarchical structure is effective for low detection limit, high sensitivity and enhanced water repellency, ${ }^{[25,35]}$ and thus was a rational design for both high sensing performance and superhydrophobic surface to expand the application scope and environments that the sensor can be used. Transmission electron microscopy (TEM) shows that the MWCNTs are uniformly dispersed in RTV and intertwined with each other (Figure 1e), contributing to the mesh-like MWCNT percolation networks. It should be noted that the MWCNT networks were completely encapsulated by RTV rather than being physically deposited or partially embedded on/in the RTV, this provided the conductive networks with strong substrate adhesion, even after multiple repeated stretching/releasing cycles, and thus is beneficial for high reproducibility and durability. Energy-dispersive spectroscopy (EDS) mapping images demonstrate only $\mathrm{C}, \mathrm{O}, \mathrm{Si}, \mathrm{S}$ elements distributed on the composites surface (Figure 1f-i), indicating that the superhydrophobic MRS material is fluorine-free, and it would greatly reduce the environmental pressure, ${ }^{[36]}$ which is further verified by the result of X-ray 
photoelectron spectroscopy (XPS) (Supplementary Figure S2, and Supplementary Note 2).

Synthesis of the multifunctional MRS material is simple at comparatively low-cost. It mainly comprises two commercial inexpensive raw materials, MWCNT $(113.0 £ / \mathrm{kg})$ and $\operatorname{RTV}\left(13.6 £ / \mathrm{kg}\right.$ ), and thus its material cost is as low as $42.0 £ / \mathrm{m}^{2}$ (see Supplementary Note 3). Simultaneously, the entire preparation procedure only requires room temperature curing and a fast sanding process (within $1.0 \mathrm{~cm}^{2} / \mathrm{s}$ ). Radar diagram was used to evaluate processing approaches to multifunctional wearable sensors. The rating system of the radar diagram was given in Supplementary Note 4. The smaller area of the radar diagram indicates more competitive for industrial production. As shown in Figure 3, (Supplementary Table S1), our approach, without the requirements for complex procedures, operation at harsh temperature, or any additional equipment, is more promising for large-scale production, compared with recently reported approaches to functional strain sensors. 


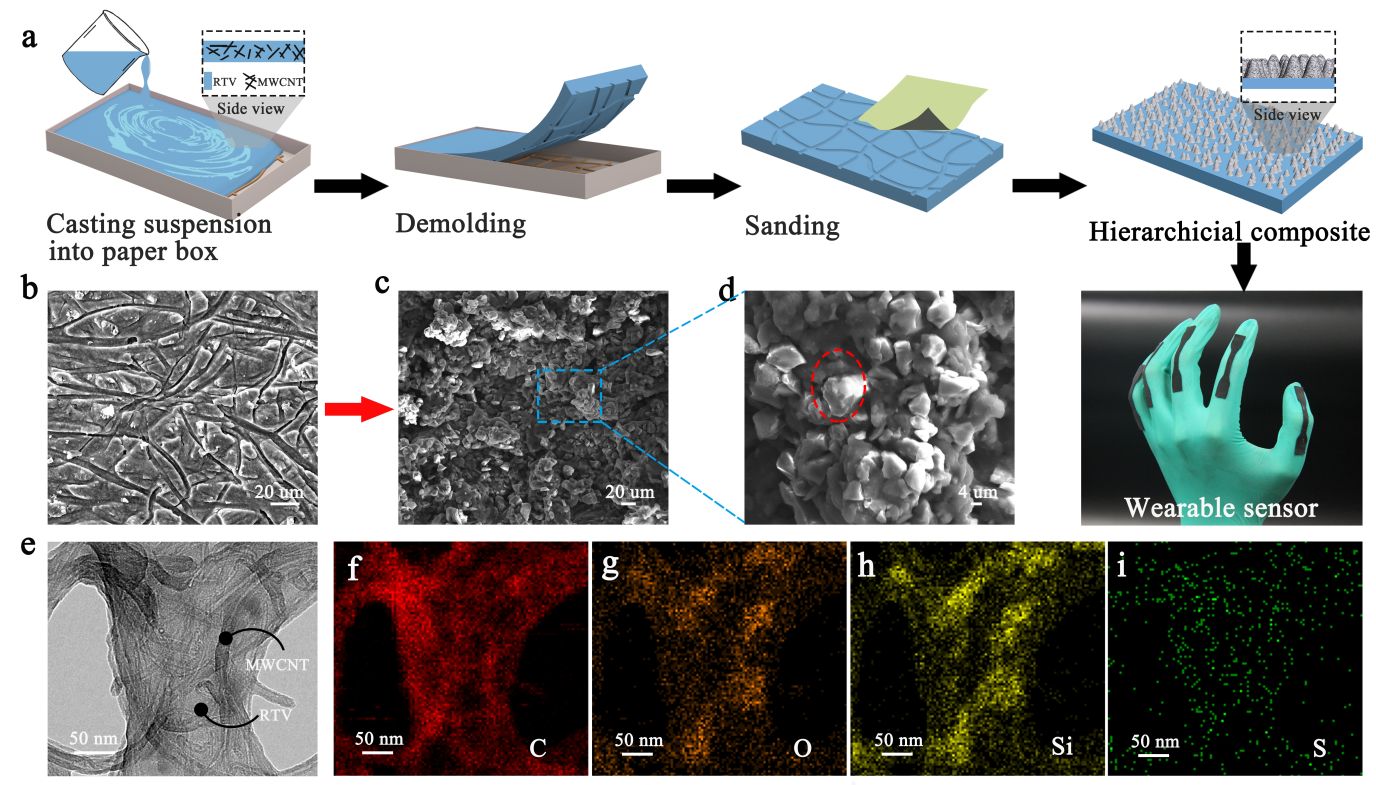

Figure 1. Preparation and characterization of Lotus leaf-inspired hierarchical and stretchable MRS material. a) Schematic illustration of the fabrication process. b-c) SEM images of the MRS material before (b) and after (c, d) sanding process. e) TEM image and (f-i) corresponding element mapping images of the MRS material.
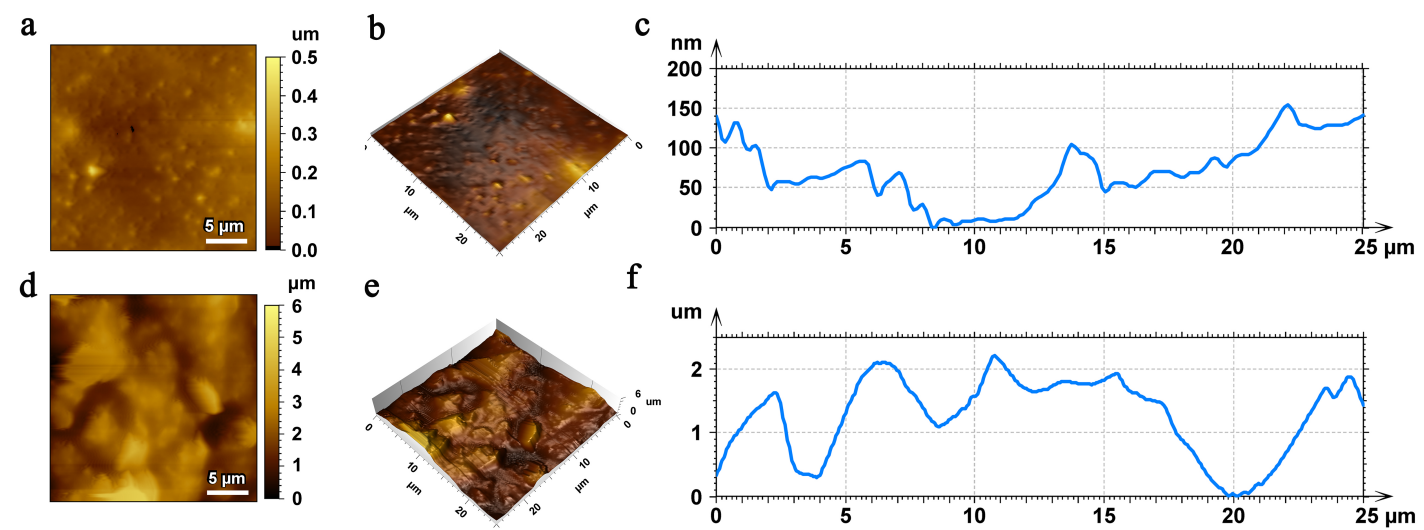

Figure 2. AFM images and roughness values of the top (a-c) and bottom (d-e) surface of the MRS material. 
a) Ref. 27

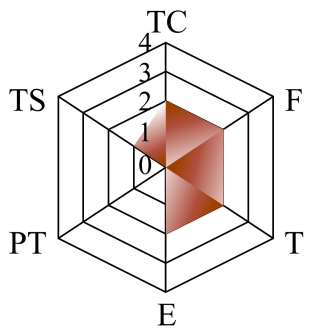

e) Ref. 22

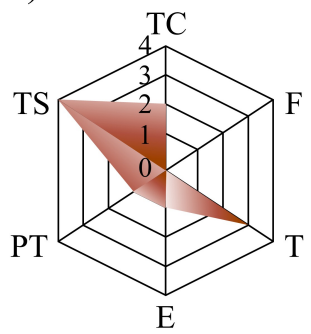

i) Ref. 25

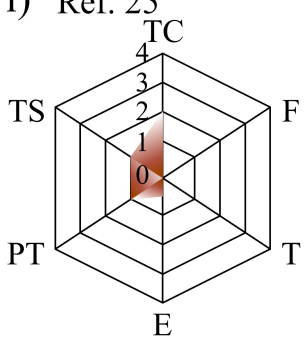

b) Ref. 24

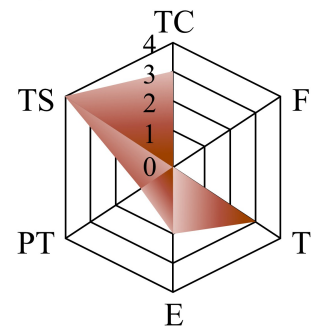

f) Ref. 29

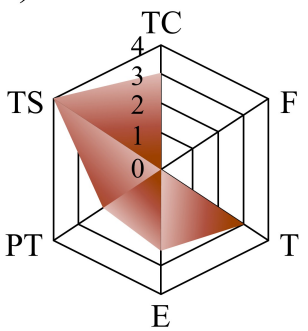

j) Ref. 26

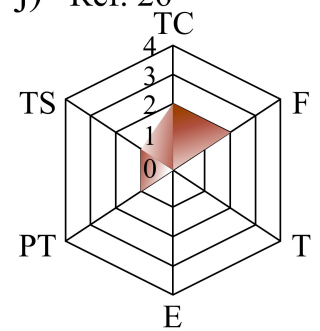

c) Ref. 19

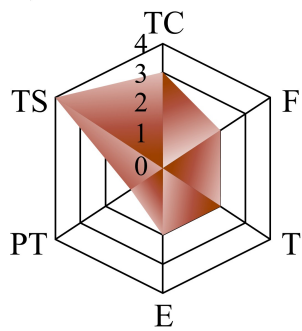

g) Ref. 23

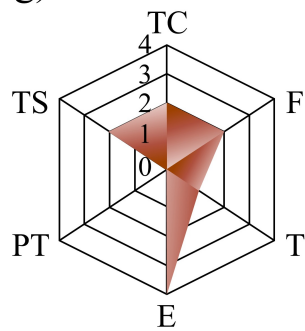

k) Ref. 31

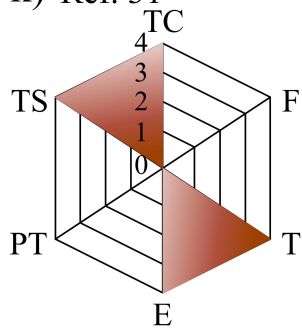

d) Ref. 28

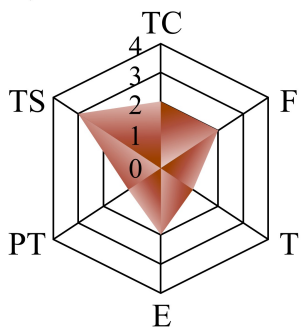

h) Ref. 30

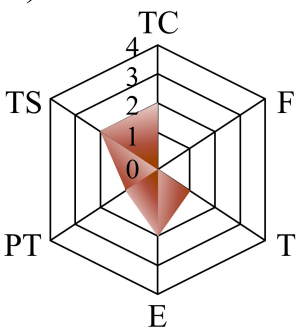

1) This work

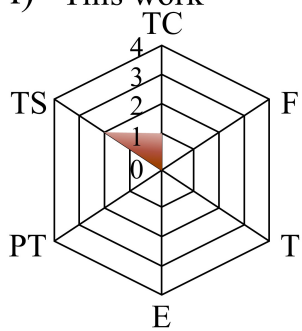

$0=$ very easy, $1=$ easy, $2=$ average, $3=$ complicated, $4=$ very complicated

Figure 3. Radar diagram of the evaluation of most common multifunctional strain sensor production methods in terms of total chemicals (TC), fluorine involvement (F), temperature requirement $(\mathrm{T})$, equipment $(\mathrm{E})$, pre/post treatment requirement $(\mathrm{PT})$, total steps (TS).

The sensing performance and practical application of the MRS material were investigated under different conditions. The following results were all obtained using the sample prepared by $10 \mathrm{~mL}$ suspension with an optimal MWCNT loading of 3.5 $\mathrm{mg} / \mathrm{mL}$ (in cyclohexane), which was determined by preparing a series of MWCNT/RTV composites using suspension with different MWCNT loadings and 
volumes and testing sensitivity and sensing range (Supplementary Note 5, Supplementary Figures S3, 4, 5). The surface conductivity and thickness of the MRS material was $0.065 \mathrm{~S} / \mathrm{m}$ and $645.3 \mu \mathrm{m}$ (Supplementary Figures S6, 7).

To investigate the strain sensing properties of the MRS materials, the resistance $(R)$ was recorded in real-time during stretching, and the relative resistance $\left(\Delta R / R_{0}\right)$ was calculated as $\Delta R / R_{0}=\left(R-R_{0}\right) / R_{0}$, where $R_{0}$ represents the film resistance at the initial relaxed state. Figure 4a shows a graph plotted of resistance versus applied tensile strains on different film types of MRS materials: hierarchically structured and unstructured. The gauge factor (GF), an important parameter to characterize strain sensitivity, was calculated by $\left(\Delta R / R_{0}\right) / \varepsilon$, where $\varepsilon$ is strain. Thus, the slope of the graph is the actual magnitude of the sensitivity or gauge factor. For both the two samples, sensitivity increased with tensile strain. However, compared with unstructured MRS material, the structured film exhibits significantly higher sensitivity at the same strain over the whole sensing range (Figure 4a). The maximum GF of the hierarchically structured MRS material was 313.7 , which is $\sim 6.4$ times higher than the maximum sensitivity of the unstructured one. These results confirm that the hierarchical structure is effective to enhance strain sensitivity. The improved sensitivity was attributed to the strain redistribution on the MRS material with hierarchical structure. For a typical stretchable strain sensor, sensitivity depends on the distance change between conductive materials. It has been reported the sensitivity could be significantly improved when the stretchable conductive materials are deposited in the 
regions where the local strain is magnified. ${ }^{[37]}$ In this case, larger distance change between conductive materials is obtained. The hierarchically structured MRS material has uneven micro-nano binary structure created by a lot of protuberances after sanding process (Figure 1c-d). Uneven microstructures on sensor surfaces are effective to achieve surface strain redistribution during deformation and thus obtain magnified local strain. ${ }^{[37]}$ However, the unstructured MRS material has homogeneous strain distribution during deformation, which is not in favor of large distance change between conductive materials. Therefore, hierarchically structured MRS material demonstrated higher sensitivity than the unstructured one. Moreover, the hierarchical structured MRS material could sense strain up to $447 \%$ while the sensing range of the unstructured sample was $407 \%$.

The structured MRS material was used as the testing sample for further study, unless otherwise specified. Based on principle of high linearity $\left(\mathrm{R}^{2}>0.99\right.$, in present work), as shown in Figure 4b, the work ranges of the sensor can be divided into six different sensing regions with average GF of $2.1(\varepsilon<71 \%), 3.3(71 \%<\varepsilon<123.5 \%), 4.8$ $(123.5 \%<\varepsilon<167.0 \%), 7.3(167.0 \%<\varepsilon<214.8 \%), 111(375.7 \%<\varepsilon<405.4 \%)$, and 214 (405.4\% $<\varepsilon<447.0 \%)$, respectively. Of note, the average GF could reach as high as 214 even within a large strain range (up to 447\%), indicating that the MRS material simultaneously demonstrates wide sensing range and high sensitivity, which makes it extremely competitive (Figure 4c). ${ }^{[22,23,25,26,28,29,30,38,39,40,41,42,43,44,45,46,47,48,49,50,51]}$ The detailed information about the comparison of GF and maximum sensing range 
between the MRS materials and other existing strain sensors is summarized in Supplementary Table S2. Moreover, the strain responses resulted from crack propagation during stretching, indicated by the SEM images of the MRS material under different strain (Supplementary Figure S8), which is in good agreement with the sensing mechanism reported among recent polymer/nanomaterials composites based stretchable strain sensors. ${ }^{[19,24,27,28]}$

To study the detection limit of the MRS materials, we used a stepper machine to gradually increase the step strain. Figure $4 d$ shows that the MRS materials can respond to extremely minute strain of $0.2 \%$ (corresponding to a relative resistance $\Delta R / R_{0}$ of $0.159 \%$ ), while the output signals were highly repeatable (inset in Figure $4 \mathrm{~d})$. However, as the strain resolution of the stepper machine is $0.2 \%$, the current method is not able to determine the detection limit of the MRS materials. We further designed a water dropping method to determine the detection limit due to the superhydrophobic properties of the MRS materials, which will be shown later in the sections regarding superhydrophobicity and practical sensing applications. Using the water dropping method, we find that the MRS materials are able to response to an extremely low strain with relative resistance of $0.024 \%$, which is far smaller than $0.159 \%$ relative resistance when the strain was $0.2 \%$, indicating that the detection limit of the MRS materials is below $0.2 \%$.

The dynamic performance of the MRS material under both small and large strains was determined by applying multiple stretching/releasing cycles at strains of $20 \%, 50 \%$, 
$100 \%, 200 \%$, 440\%, respectively. As shown in Figure 4e, the signal shape and intensity (i.e., the maximum of $\Delta R / R_{0}$ ) of each response was almost identical at a fixed strain, demonstrating a highly reliable and stable response over a wide sensing range. The frequency-independent sensing property, one of the major characteristics in the sensor fields, ${ }^{[25]}$ was studied by repetitively applying a $50 \%$ strain at different frequencies on the sensor. The result shows that the MRS material was capable of working at different stretching frequencies from 0.1 to $2 \mathrm{~Hz}$ with high reproducibility (Figure 4f), which benefits from its fast response rate (response time was less than $122.67 \mathrm{~ms}$, Supplementary Figure S9, Supplementary Note 6). It should be noted that the first deformation of the MRS material during cyclic stretching is irreversible (Supplementary Figure S10). Before starting cyclic stretching, $\mathrm{R}_{0}$ was recorded and one irreversible cycle was conducted on the MRS material and thus resulting in the $\Delta R / R_{0}$ value shown in Figure $4 \mathrm{~d}-\mathrm{g}$ did not start from 0 (More details see Supplementary Note 7).

The dynamic durability of the MRS material was determined by applying cyclic stretching from $\varepsilon=0 \%$ to $\varepsilon=50 \%$ for more than 10000 cycles, and the result is shown in Figure 4g. Although there was a small drop in relative resistance in the initial cycles, the response to periodic strain became stable after 300 cycles. This is because MWCNT networks were stabilizing their microstructure during the damagereconstruction processes during initial cycles. ${ }^{[52]}$ The inset in Figure $4 \mathrm{~g}$ shows the close-up of 40 cycles that were randomly extracted from the 10000 cycles, the signal 
shape and intensity of each cycle had no apparent change, thus indicating the high stability and durability of the MRS material. For convenience of comparison, the dynamic durability was further tested by applying periodic strain (from 0 to 200\%) on the sensor for 5500 cycles, and the results show that the sensing performance was not impaired during the cyclic stretching process at such large strain (Supplementary Figure S11). It should be noted that the dynamic durability in the present work is superior compared with existing superhydrophobic strain sensors to the best of our knowledge (Figure $4 \mathrm{~h}$ and Supplementary Table S3). ${ }^{[23,24,25,26,27,28,29,30,53]}$ The high dynamic durability of the MRS material is due to the following reasons: 1) the MWCNT networks were completely embedded into RTV substrate, hence the RTV substrate provided strong binding strength to MWCNT networks, which prevented conductive networks sliding away from RTV under dynamic loading. ${ }^{[54]}$ 2) High elastic behavior and long length (up to $50 \mu \mathrm{m}$ ) of MWCNTs avoid plastic deformation and fracture of the conductive networks during multiple stretching processes, even at large strain. ${ }^{[32]}$ 3) Hierarchical structure is helpful for external stress release, ${ }^{[55]}$ aiding the MRS material to withstand repeated large strain without affecting its stretchability.

Besides stretching, bending and torsion are another ubiquitous mechanical deformation. To realize the detection of bending and torsion, the MRS material was integrated on a hot melt glue stick with one end fixed and the other end bent or twisted (detailed measurement seen Supplementary Figure S12). Bending and torsion induced strain on the MRS material, which served as stimulus to the sensor and in 
turn reflected the bending and torsion extent. The MRS material exhibited a wide sensing range up to a bending of $140^{\circ}$ and a torsion of $628 \mathrm{rad} \mathrm{m}^{-1}$ with high reproducibility (Figure 5).
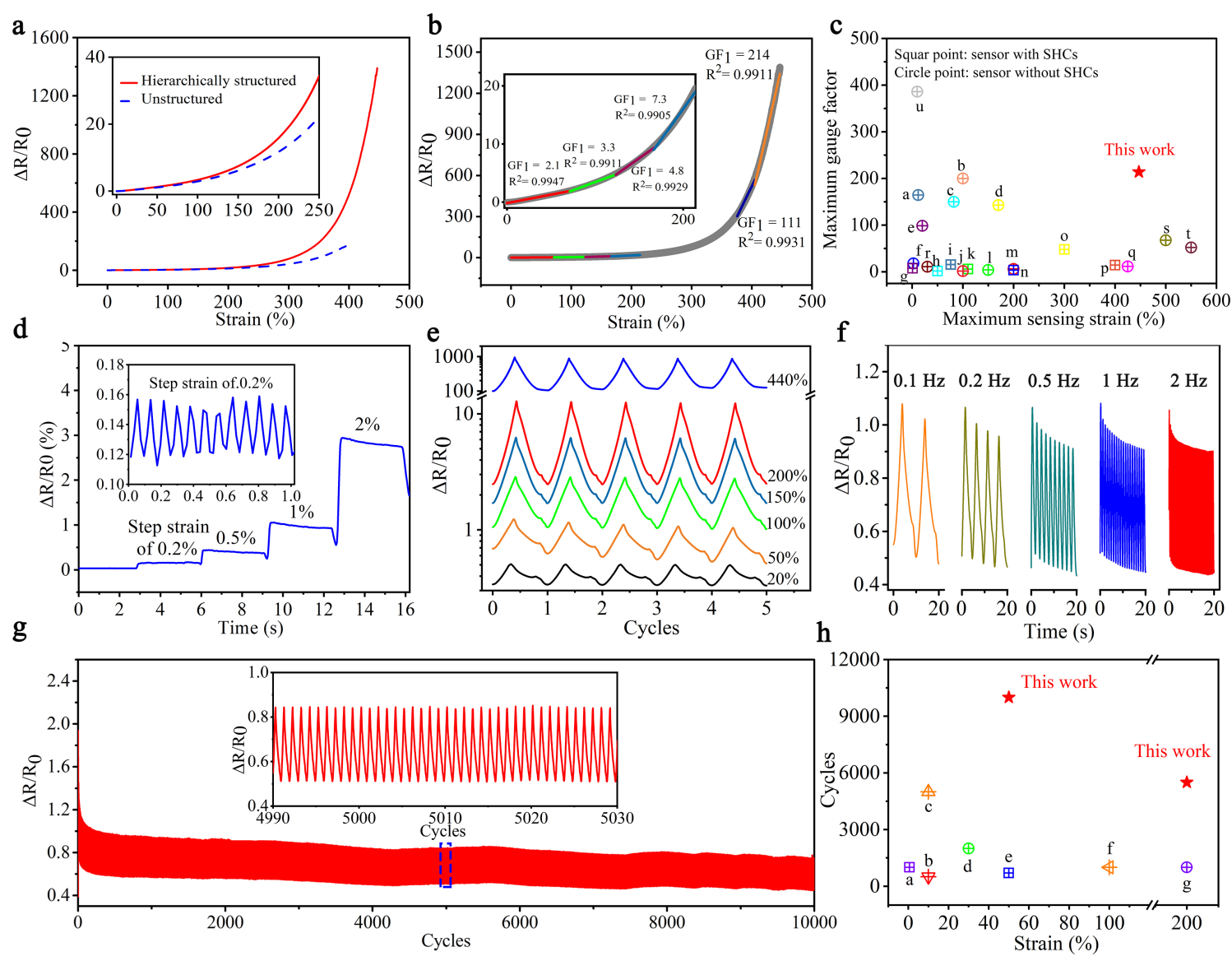

Figure 4. Sensing performance and dynamic durability of the MRS material towards stretching. a) Relative resistance as a function of tensile strain applied on different MRS material types: hierarchical structured (red solid line) and unstructured (blue dashed line). b) The linear fittings of the hierarchically structured MRS material. c) Comparison of maximum gauge factor-maximum sensing range in reference and that in our work. a-u corresponding to refs $41,47,39,44,42,38,30,29,25,45,28,40$, $48,23,22,26,46,43,49,50,51$, respectively. SHCs represents superhydrophobic 
surfaces d) Relative resistance under gradually increasing step strain from $0.2 \%$ to 2\%. Inset: Relative resistance during 12 stretching cycles at $0.2 \%$ strain. e) Relative resistance during five stretching cycles at a fixed strain from $20 \%$ to $440 \%$. f) Realtime relative resistance under repetitive stretching from 0 to $50 \%$ strain with different frequencies. g) Relative resistance under cyclic stretching from 0 to $50 \%$ strain over 10000 cycles. h) Comparison of dynamic durability in reference and that in our work. a-g corresponding to refs $30,27,25,24,53,28,23$, respectively.
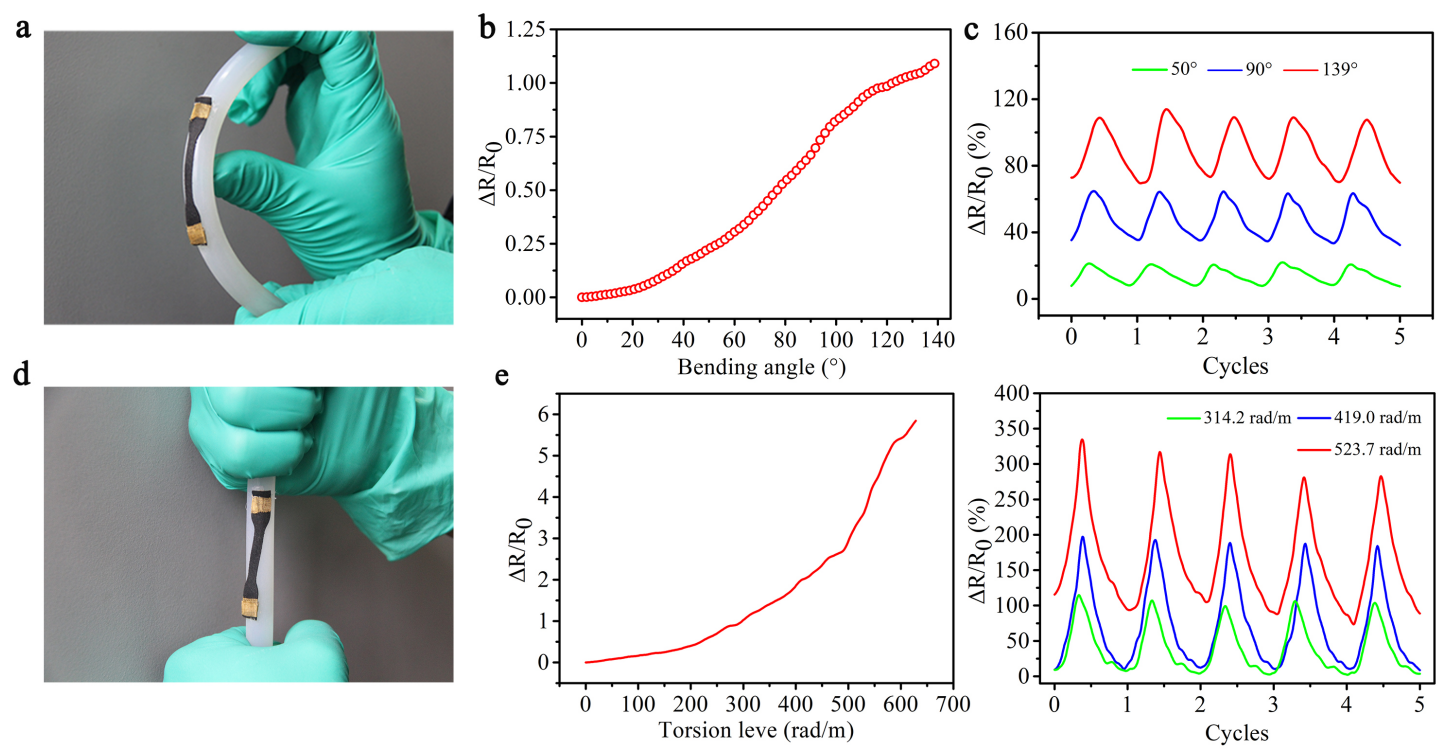

Figure 5. Sensing performance and dynamic durability of the MRS material towards bending/torsion. a) Illustration of the sensor at bending state. b) Relative resistance variation as a function of bending angle. c) Real-time variation in the relative resistance under repetitive bending at different fixed angles. d) Illustration of the sensor at torsion state. e) Relative resistance variation as a function of torsion angle. f) Real-time variation in the relative resistance under repetitive torsion at different fixed angles. 


\subsection{Superhydrophobicity and multifaceted robustness of the coating-free MRS}

materials

To retain a high degree of the above-mentioned sensing performance (i.e. high sensitivity, wide sensing range and robust dynamic durability), we designed this sensing material to be superhydrophobic by creating surface rough morphology to avoid the need for a water-proof coating. The superhydrophobic MRS materials were designed to function particularly in harsh environments. For example, when they are applied as flexible and stretchable sensors, their superhydrophobicity are expected to be robust towards various mechanical deformation and destruction, and chemical corrosion etc. Here, controllable water repellency and multifaceted robustness is studied.

The water contact angles (WCAs) of the top and bottom surfaces of the MRS material were $104.0^{\circ}$ and $118.1^{\circ}$, respectively (Supplementary Figure S13a and c), indicating its hydrophobicity. We simply rubbed the MRS material with sandpaper to create rough surface morphology, then both sides became superhydrophobic with water contact angles of $150.6^{\circ}$ and $154.5^{\circ}$, respectively (Supplementary Figure S13b and d, Supplementary Movie S1, and Figure 6a). It should be noted that chemical modification to lower surface energy was not required due to the intrinsic hydrophobicity of RTV. The operation requirements for achieving superhydrophobicity were comprehensively studied. We first studied the influence of abrasion direction on surface morphology and wettability. SEM images show that 
unidirectional abrasion direction induced parallel long grooves, orthogonal abrasion direction produced vertically intersecting long grooves, while no long grooves were observed on the surface rubbed along random directions (Supplementary Figure S14). Nevertheless, all these obtained surfaces show superhydrophobicity with static water contact angles of beyond $154.0^{\circ}$ and sliding angles (SAs) of $\sim 6.0^{\circ}$, indicating a slippery Cassie-Baxter type surface (Figure 6b). These results verify that abrasion direction is not a key factor that determines surface wettability although it has an impact on surface morphology. Additionally, a wide range of types of abrasive paper can be used to texture the superhydrophobic structure. As shown in Figure 6c, WCAs over $150.0^{\circ}$ and SAs below $10.0^{\circ}$ were obtained on the surfaces after abrasion using sandpapers with grit No. ranging from 80 to $1000 \mathrm{Cw}$. The surfaces rubbed by 80,100 and $180 \mathrm{Cw}$ abrasive papers had water contact angles as high as $\sim 154.0^{\circ}$. It was also found that the water contact angles of the resultant surfaces showed a decrease tendency with the increase of grit No, which is attributed to the surface structure with decreased roughness (Figure 6d-f, details seen Supplementary Note 8). Abrasive paper with smaller grit No. provides greater pressure and frictional resistance and thus is favor of higher surface roughness achievement. Moreover, the superhydrophobicity of the MRS material did not depend on MWCNT loading or volume of suspension used (Supplementary Figure S15 and Supplementary Movie S2), this is because the RTV films could achieve superhydrophobicity after being textured with hierarchical structures (Supplementary Figure S16). Superhydrophobicity of the MRS material 
was achieved at both relaxed and tensile states (Supplementary Figure S17). Obviously, it does not require professional operating skills, precise control in preparation process or expensive equipment/materials to obtain the superhydrophobic MRS material. Without specific notification, superhydrophobic MRS materials were used in different tests and were fabricated through being randomly rubbed by $180 \mathrm{Cw}$ sandpaper at a relaxed state.

Mechanical durability of sensing materials is highly significant for their practical applications particularly on flexible and wearable strain sensors, however, superhydrophobic materials are usually mechanically weak; ${ }^{[33]}$ it is challenging to fabricate a mechanically robust and stretchable strain sensor with superhydrophobic properties. Figure 7 a shows that a water droplet retained its spherical shape on the stretched composite surface at strain of 0 and $450 \%$, and in both cases the WCAs were beyond $\sim 154^{\circ}$ while SAs were lower than $8^{\circ}$. Additionally, when water droplets drop onto the MRS surfaces during the stretching process, they readily rolled off (Supplementary Movie S3). Figure 7b shows the superhydrophobic MRS surfaces retained a WCA of $151.0^{\circ}$ and a sliding angle lower than $10.0^{\circ}$ after 5000 cycles of stretching/releasing processes at $200 \%$ strain. The superhydrophobic stability and durability of the MRS materials towards bending deformation was further tested and it showed that superhydrophobicity was retained with an increasing bending angle up to $180^{\circ}$ and after 5000 bending cycles at fixed angle of $180^{\circ}$ (Figure $7 \mathrm{c}-\mathrm{d}$ ). These results confirm that the superhydrophobicity of the MRS materials is highly stable towards 
dynamic, large and long-term strain, which ensures the waterproofing protection to function over the whole sensing range and after repeated sensing cycles. To further test its superhydrophobic durability, we performed various mechanical tests including multi-cycle abrasion, finger-wipe, tape-peeling, knife-scratc (Figures 7e-h, Supplementary Figure S18-19 and details seen Supplementary Note 9). The MRS materials retained their superhydrophobicitiy after all these tests.

The superior robustness can be explained by the following reasons: 1) RTV is an elastic material, which shows a more desirable anti-abrasion ability than a rigid one. ${ }^{[33]}$ This is because elastic material can largely dissipate the shear force produced by friction into the whole surface rather than the local abraded area. 2) The hierarchical structure in support of superhydrophobicity was generated from the sanding process. Consequently, the surface micro-nano structure would be further engineered rather than destroyed during sandpaper abrasion test. 3) Our raw material selection enables low surface energy material throughout the bulk material rather than being confined on the surface, ${ }^{[56]}$ thus providing sufficient hydrophobic material to resist sandpaper abrasion and imparting the MRS material with excellent chemical and environmental durability.

Besides mechanical abrasion, stable water repellency in other harsh conditions, such as corrosive liquids, high temperature, UV irradiation and high humidity, are also important for consideration of practical applications of the sensing materials. We further performed soak tests in aqueous solution with $\mathrm{pH}$ ranging from 1 to 13 for 12 
$\mathrm{h}$, high-temperature environmental tests $\left(250^{\circ} \mathrm{C}\right)$ for $76 \mathrm{~h}$, UV exposure for $30 \mathrm{~h}$ and high-humidity environmental tests $\left(90 \%, 35^{\circ} \mathrm{C}\right)$ for $80 \mathrm{~h}$ (Supplementary Figure S20, Supplementary Movie S4 and details seen Supplementary Note 10). The resultant MRS materials retained superhydrophobicity after all the above-mentioned damages, indicating that the superhydrophobic MRS materials are promising to be applied under various harsh environmental conditions.

a
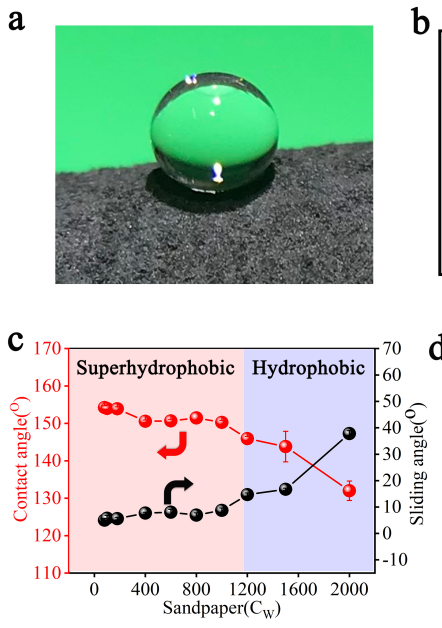
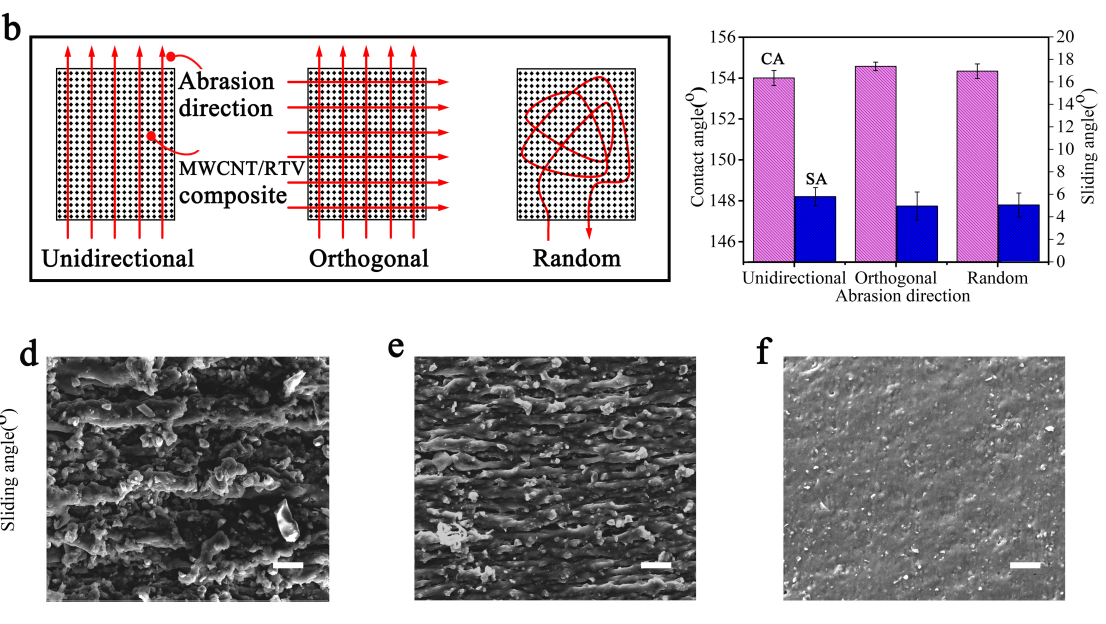
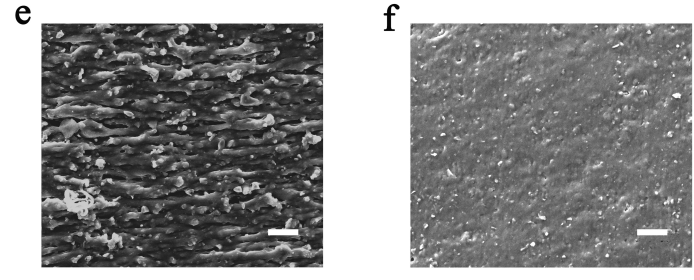

Figure 6. Controllable fabrication of the superhydrophobic MRS material. a) A water droplet beaded up on the MRS surface. b) Water contact angle and sliding angle as a function of abrasion direction. c) Water contact angle and sliding angle dependent of abrasive sandpapers with grit No. from $80 \mathrm{Cw}$ to $2000 \mathrm{Cw}$. d-f) SEM images of the MRS surfaces that were rubbed by different sandpapers with grit No. $400 \mathrm{Cw}$ (d), $1200 \mathrm{Cw}$ (e) and $2000 \mathrm{Cw}$ (f). Scale bars, $40 \mu \mathrm{m}$. 

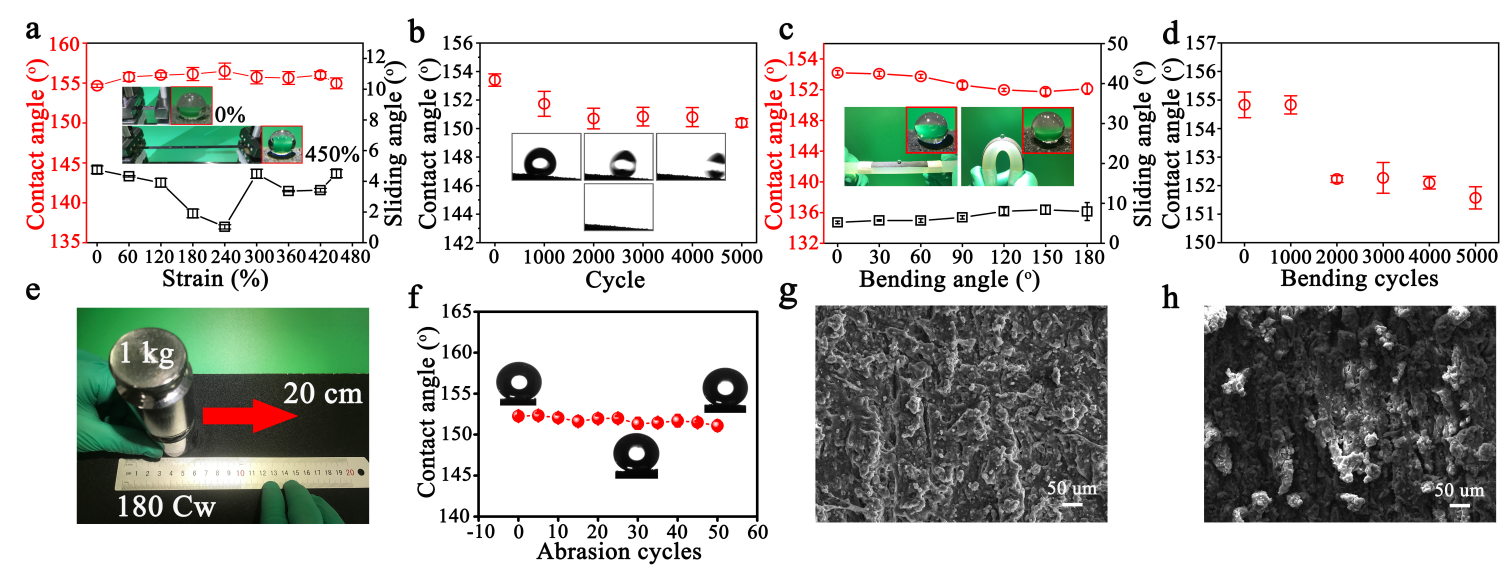

h

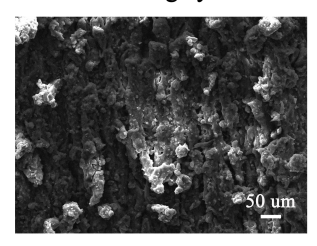

Figure 7. Multifaceted robustness characterization of the superhydrophobic MRS

material. a) Water contact angle and sliding angle variation during stretching the MRS materials stretched from 0 to $450 \%$ strain. Insets: spherical water droplets on the MRS surface at strain of 0 and $450 \%$. b) Water contact angles of the MRS materials after 0 5000 stretching cycles at $200 \%$ strain. Inset: Water droplet sliding away from the composites surface at a slope angle within $10^{\circ}$. c) Water contact angle and sliding angle variation during the MRS materials bent from 0 to $180^{\circ}$. Insets: spherical water droplets on the MRS surface with bending angle of 0 and $450 \%$. d) Water contact angles of the MRS materials after $0-5000$ bending cycles at an angle of $180^{\circ}$. e) Schematic illustration of abrasion test. f) Water contact angle as a function of abrasion cycles. Insets: Optical photographs of water droplet on the MRS materials after 0, 25, 50 abrasion cycles. g-h) SEM images of the MRS surfaces before (c) and after 50 abrasion cycles.

\subsection{Sensing performance in different harsh environments}

Given superhydrophobic properties, sensing materials are able to function in harsh 
environments such as water, sweat, acid and alkali without any coatings. ${ }^{[19,22,23,24 \text {, }}$ 25,26,27,28,29,30] Nevertheless, the protection performance in other rigorous conditions such as dirt, high humidity and high temperature has not been reported. Here, the stability and reliability of the superhydrophobic MRS materials under various environments were comprehensively studied. To compare with superhydrophobic MRS materials, we also performed the same tests on MRS samples before sanding treatments - these samples are not superhydrophobic but have a medium degree of hydrophobicity.

The relative resistance changes of two different MRS material types were measured when in contacted with water while sensing, under static and dynamic stretching/releasing states (strain from 0 to $50 \%$ ). In the case of the MRS material without superhydrophobicity, $\Delta R / R_{0}$ value decreased immediately and dramatically when its surfaces contacted water, even without deformation (Figure 8a). This is because water adhered on the MRS surface and affected the conductive MWCNT networks (via changing charge pathways). During stretching/relaxing cycles, as shown in Figure $8 \mathrm{~b}, \Delta R / R_{0}$ of the unstructured MRS material varied periodically before water dropping. Once water dropped on its surface, the output signal rapidly changed both in $\Delta R / R_{0}$ value and shape. Such changes were complex and show no regularity, as a result of dynamic water dispersion on the MRS surface during the stretching/relaxing process (Figure 8c). Moreover, even stopping water dropping, the maximum $\Delta R / R_{0}$ of each cycle could not recover to the original value. The resultant 
$\Delta R / R_{0}$ value varies among repeated tests due to the different amounts and dispersion location of water left on the MRS surface (Supplementary Figure S21). As such, calibration could not be taken to correct the unreliable signal output during or after raining. These results confirm water has a significant effect on reliable sensing performance of the MRS material without superhydrophobicity. However, when water contacted with the superhydrophobic MRS materials at static states, it quickly rolled off due to low water adhesion on the superhydrophobic MRS surface, and thus its sensing performance was stable $\left(\Delta R / R_{0}=0\right)$ (Figure $\left.8 \mathrm{~d}\right)$. When water was dropped onto the superhydrophobic MRS materials during the cyclic stretching process, it demonstrated unaffected cyclic changes in relative resistance (Figure 8e-f), indicative of its stable and reliable sensing performance under rainy (or sweat-like) conditions.

Superhydrophobicity could protect MRS materials not only from water, but also from strong acid and alkali, artificial sweat and daily liquid contaminates e.g., coffee and soy sauce (Figure 8g, Supplementary Figure S22). More impressively, such protection is effective at high temperature (up to $60{ }^{\circ} \mathrm{C}$ ) and high humidity $(91 \%)$ due to the robust superhydrophobicity (Figure $8 \mathrm{~h}-\mathrm{i}$ ), which has not been reported previously. This indicates that the superhydrophobic MRS materials are highly promising to function in extreme environments. 

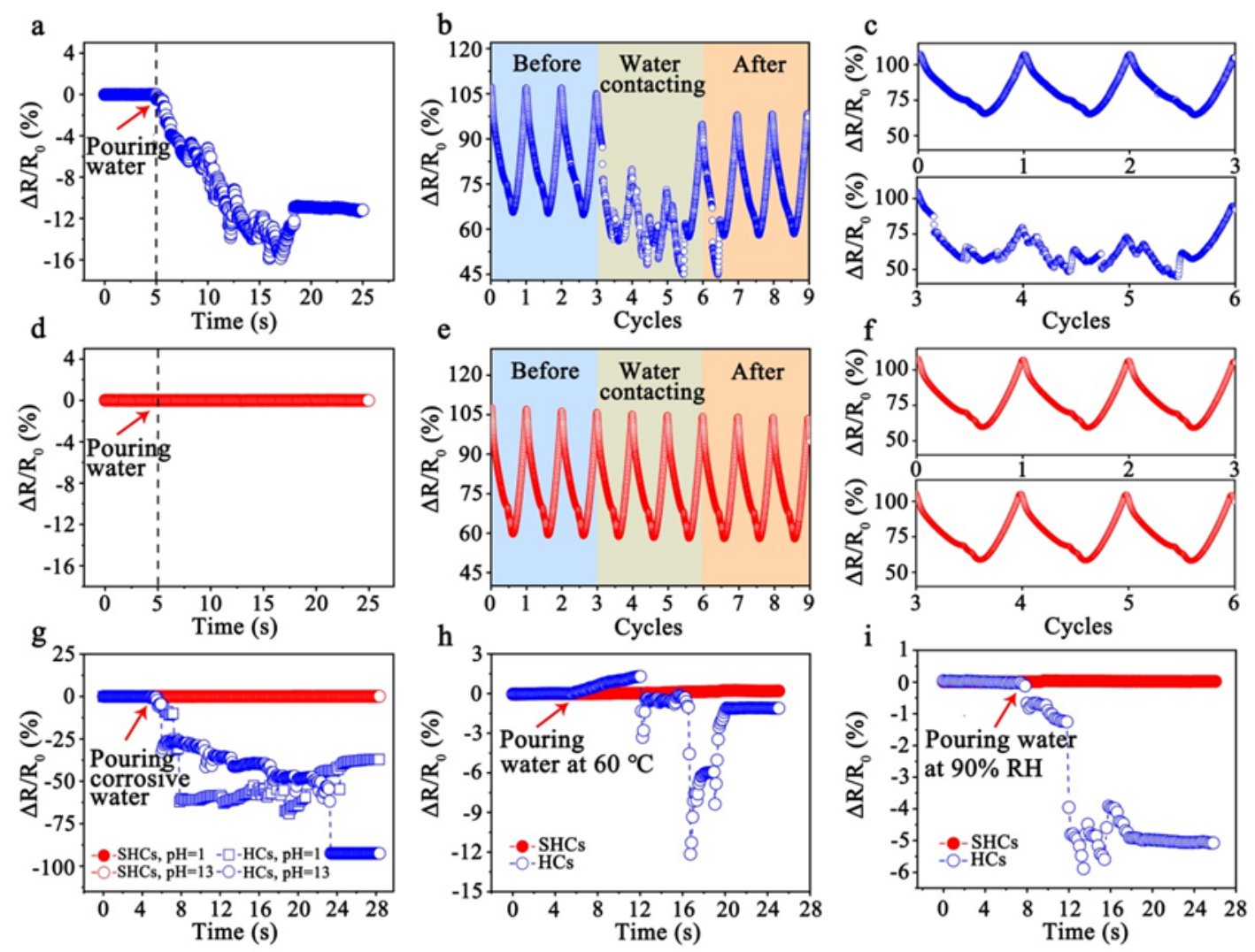

Figure 8. Sensing performances of different types of MRS material under various harsh environments. a-c) Relative resistance changes of MRS material (without sanding treatments) before and after water dropping under static state (a) and dynamic stretching/relaxing state (strain from 0 to $50 \%$ (b). c) Enlarged image of (b). d-f) Relative resistance changes of the superhydrophobic MRS material before and after water dropping at static state (d) and dynamic stretching/relaxing state (strain from 0 to $50 \%$ (e). f) Enlarged image of (e). g-i) Relative resistance changes of unabraded and superhydrophobic MRS materials at static states under different vigorous conditions: corrosive water $(\mathrm{g})$, temperature of $60^{\circ} \mathrm{C}$, and (h) high humidity of $91 \%$ (i). SHCs: MRS material with superhydrophobic surfaces. HCs: MRS material without sanding treatment (showing hydrophobic surfaces). 


\subsection{Practical applications: Full-range human motion and physiological detection,}

and water impact sensing

Owing to simultaneous demonstration of an impressive detection limit, sensitivity, sensing range and durability, the superhydrophobic MWCNT/RTV composite based wearable (SMRCW) sensor is capable of detecting a full range of human motions. The SMRCW sensor could be attached comfortably to different positions of the human body/skin using medical tapes due as it is ultra-lightweight (ca. $0.07 \mathrm{~g} \mathrm{~cm}^{-1}$ ) and has extreme flexibility. To capture rapid activity, the sensors were fixed to different joints including wrist, finger and knuckle (insets in Figure 9a-c), as shown in Figure 9a-c and Supplementary Movie S5. The movements of these joints could be qualitatively and clearly recognized based on the distinctly differentiated frequency (demonstrated by different times spent on 8 cyclic movements) and response curve output from the sensor. Moreover, the responses to vigorous motions were highly repeatable, verified by the almost unchanged signal shape and maximum value of each cycle. Outdoor human activities pose high demand on the strain sensor because of the complex environments such as rain. The SMRCW sensor can work under wet or rainy conditions with assistance of superhydrophobicity, which was verified by the reliable and stable response to joint movement under intermittent water dropping (Supplementary Figure S23). In minute motion sensing, the sensors were attached to the fingertip and throat (insets in Figure 9d-e). Blood pulse rate could be read out easily from a static fingertip. Figure 9d and Supplementary Movie S6 show that the 
sensor could capture the amplitude and frequency of pulse from the fingertip via blood periodically flowing (each cycle denotes one pulse). In Figure 9e, when the tester spoken "Hi", "Hello", "Sensor", "Superhydrophobic" and "Environment", the sensor outputted distinguishable signals for each word with good reproducibility (Supplementary Figure S24, Supplementary Movie S7), as a result of deferent vocal cord movements. As such, the present sensor is promising to be used for many applications such as human-machine interface, personal health management and speech rehabilitation training.

A water droplet impinging onto a superhydrophobic surface bounces off, similar to a football hitting the ground. ${ }^{[57]}$ When water bouncing occurs on the SMRCW sensors, it causes an impact force that acts on the sensor and thus arouses a resistance change. In this way, SMRCW sensors are potentially capable of sensing water droplets, which has many promising applications e.g., measurement platform (examples of evaluation of superhydrophobicity and agricultural sprinkler irrigation system) and rainfall monitoring. Additionally, when superhydrophobic surfaces protect sensors from rain, it is necessary to calibrate the sensors to compensate for the influence of water impact. However, few studies have been reported among existing superhydrophobic strain sensors. Here, the sensing performance to water droplets was studied by releasing droplets from a certain distance above the sensor, which was fixed on a sloping platform with an angle of $8^{\circ}$ (schematic diagram is given in Supplementary Figure S25). The resistance of the sensor was recorded in real time during the water 
bouncing process. As shown in Figure 9g, when a droplet of $50 \mu \mathrm{L}$ was dropped on the SMRCW sensor surface from a height of $10 \mathrm{~cm}, \Delta R / R_{0}$ increases immediately and then reaches the maximum value of $0.123 \%$. Once the droplet rebounded off the surface, $\Delta R / R_{0}$ recovered quickly and completely. Such response is highly reproducible, confirmed by the cyclic change of $\Delta R / R_{0}$ to the consecutive water bouncing. Stable and repeatable response to water bouncing was further verified by $50 \mu \mathrm{L}$ droplets falling from $5 \mathrm{~cm}$ height (Supplementary Movie S8). Moreover, the number of consecutive droplets can be read by counting peaks in Figure 9g and Movie S8. To study the sensing mechanism, a free fall water droplet impacted on the SMRCW sensor surface was recorded by a high-speed camera. The resultant water droplet impacted the sensor surface and then rebounded off (Figure 9f). The mechanical deformation of the SMRCW sensor occurred during the collision process, and then completely recovered after the droplet rebounded and slid away from the sensor surface (Supplementary Movie S9), which indicates water bouncing makes the sensor generate a recoverable strain and thus be able to respond to continuous water droplets.

The relationship between $\Delta R / R_{0}$ and drop height, mass (here, represented by volume) was respectively measured and presented in Figure 9h-i. Each point is reported as the average of more than 5 repeated water bouncing tests. In Figure $9 \mathrm{~h}, \Delta R / R_{0}$ increased monotonically with drop height ranging from 2 to $15 \mathrm{~cm}$ (all droplets with volume of $50 \mu \mathrm{L})$ and the fitting demonstrates a good linear relationship between these two 
parameters $\left(\mathrm{R}^{2}\right.$ of 0.9962$)$. It is worth noted that a $50 \mu \mathrm{L}$ free fall water droplet with drop height of $15 \mathrm{~cm}$ results in an impact of $1.73 \mathrm{~m} \mathrm{~s}^{-1}$ on the sensor that could easily destroy the superhydrophobicity (detailed information is given in Supplementary Note 11), however, it is stable due to the impressive superhydrophobic robustness of the SMRCW sensor. Figure 9i shows that the sensor is capable of discriminating water droplets with different volumes from $20 \mu \mathrm{L}$ to $60 \mu \mathrm{L}$, and a linear fitting of these two sets of data gives $\mathrm{R}^{2}$ value of 0.9732 . These results confirm a broad sensing range towards height and mass when the SMRCW sensor is used for sensing droplets impact. Additionally, the fitting curve with high $\mathrm{R}^{2}$ value demonstrates a promising application in predicting the position and mass of droplets.

It is worthy to note that $50 \mu \mathrm{L}$ water droplet (drop from a height of $10 \mathrm{~cm}$ ) resulted in a relative resistance $\Delta R / R_{0}$ of $0.123 \%$ (Figure $9 \mathrm{~g}$ ), smaller than $0.2 \%$ strain - induced relative resistance change with $\Delta R / R_{0}$ value of $0.159 \%$. For a small strain region $(\varepsilon<$ $71 \%$ ), the relative resistance change and strain demonstrated a high linear relationship with $\mathrm{R}^{2}$ of 0.9947 . Therefore, $50 \mu \mathrm{L}$ water impact-induced strain is smaller than $0.2 \%$. More impressively, the sensor can even response to a $20 \mu \mathrm{L}$ water droplet (drop from a height of $10 \mathrm{~cm}$ ) with relative resistance change of $0.024 \%$ (Figure $9 \mathrm{i}$ ). This is about 1/7 times smaller than $0.2 \%$ strain induced relative resistance change, further indicating the sensor is capable of sensing strain of below $0.2 \%$. 

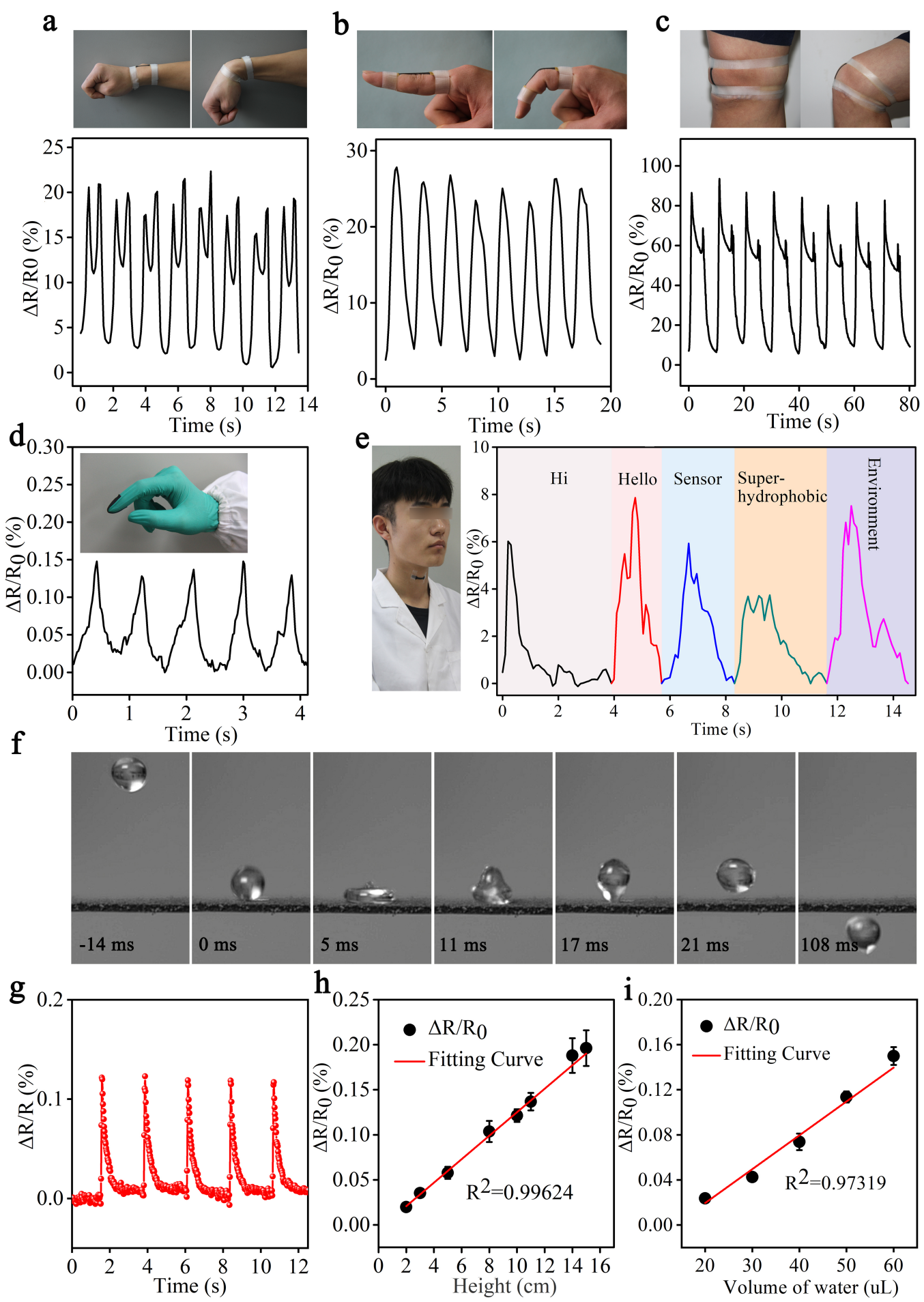

Figure 9. Practical sensing applications: Full-range human motion and physiological detection, and water impact sensing. a-c) Response signal of SMRCW sensor in monitoring activity including wrist (a), finger (b) and knuckle (c) movements, and 
corresponding sensor attached body parts. d, e) Responsive curves of SMRCW sensor in detecting tiny physiological phenomenon behaviors including pulse rate (d) and speech (e), and corresponding sensor attached body parts. f) Water bouncing on the hierarchical SMRCW sensor surface. g-i) Response signals as water bouncing sensing platform: resistance change during consecutive $50 \mu \mathrm{L}$ free-fall water droplets with drop height of $10 \mathrm{~cm}(\mathrm{~g})$, resistance changes as a function of water drop height $\mathrm{h}$ ) and water volume (i).

\section{CONCLUSIONS}

In summary, we have developed a coating-free, non-fluorinated superhydrophobic strain sensing material through homogeneously encapsulating multi-walled carbon nanotubes in room temperature vulcanized silicone rubber, followed by a roughening process. This sensing material includes multiple features such as robust dynamic durability (over 10000 stretching cycles at 50\% strain), high sensitivity (GF of 2.1 to 214), wide sensing range (up to $447 \%$ strain) and durable superhydrophobicity (mechanically, chemically, thermally, and UV impervious) in a single system. The combination of these features enables it to function even under harsh environments such as high temperature, high humidity and exposure to highly corrosive liquids. Owing to its good flexibility, high sensitivity and wide sensing range, this material is able to be used as a wearable sensor to detect full-range human motions such as wrist, finger and knuckle movements, and even small responses from physiological behavior such as pulse rate and speech recognition. Surprisingly this superhydrophobic sensor 
can detect water droplet impact/bouncing phenomena, which confirms its promising applications even in rainy conditions and further deduces its detectable strain limit is smaller than $0.2 \%$.

\section{Experimental section}

4.1 MWCNT-based suspension preparation: MWCNTs with an average diameter of 8-15 $\mathrm{nm}$ and an average length of $30-50 \mu \mathrm{m}$ were bought from Timesnano Co., Ltd., China. The RTV was purchased from Guangzhou Yiqi Trade Co., Ltd., China. The cyclohexane was purchased from Sinopharm Chemical Reagent Co., Ltd., China. All chemicals were used as received. First, MWCNTs were added into $20 \mathrm{~mL}$ of cyclohexane under magnetically stirring, followed by dispersion of $5 \mathrm{~g}$ of RTV and $0.1 \mathrm{~g}$ of curing agent. The MWCNT loading was varied from 2.5 to 6.5 milligram per milliliter of cyclohexane. Then the mixture was subjected to strong sonication (300 W) for 1 min using an ultrasonic cell disruptor (JY 99-IIDN, Ningbo Scientz Biotechnology Co., Ltd., China) and magnetically stirred for $2 \mathrm{~h}$, until a homogeneous suspension was obtained.

4.2 Hierarchical MRS material preparation: MRS material were obtained by pouring the suspension with volume ranging from 5 to $25 \mathrm{~mL}$ into a homemade mold, followed by drying at ambient conditions typically $20 \pm 1{ }^{\circ} \mathrm{C}$ and relative humidity of $80 \pm 3 \%$. Anything that is capable of holding liquids can be used as mold. Here, we chose handing fold paper box of size $7.4 \mathrm{~cm} \times 2.6 \mathrm{~cm}$ as a mold because its rough structure can be printed on the MRS material surface, favoring the generation of 
hierarchical roughness. After peeling off from mold, the MRS material at different tensile state (strain from 0 to $150 \%$ ) was abraded by different tapes of sandpaper (grit. No. from 80 to1200 $\mathrm{Cw}$ ) along different directions (including unidirectional, orthogonal and random directions) for fabrication of the hierarchical structure. Without special mention, samples $3.5-10$ were rubbed by 180 mesh sandpaper at a relaxed state along a random direction.

4.3 Characterization: The SEM images were obtained using a FEI Quanta 450 instrument at an accelerating voltage of $10 \mathrm{kV}$. The TEM images were captured using a JEOL JEM 2100F instrument at an accelerating voltage of $200 \mathrm{kV}$. The XPS images were recorded using a Thermo Scientific K-alpha photoelectron spectrometer. AFM images were collected on a Keysight 5600LS. WCAs and SAs were measured by using Data Physics OCA15 Instrument. The average of five positions was reported as the final value. For more details about wettability measurement see Supplementary Experiments. A digital camera (Canon EOS 70D) and high speed camera (fps1000HD) was used to take optical photographs and videos in this work, respectively. A stepper machine (Anka transmission technology co., ltd., China) was used to stretch the MRS material (size of $30 \mathrm{~mm} \times 10 \mathrm{~mm}, L_{0} \times W_{0}$ ) at a speed of 5 $\mathrm{mm} / \mathrm{s}$, without specific notification. Resistance measurement was conducted by connecting the two ends of MRS material to a digital source meter (Keithley 2450 SourceMeter) to read real-time electric current $\left(I_{0}\right)$, with conductive copper wires. Before tests, two ends of the MRS material were fixed to stepper machine. A constant 
voltage $\left(V_{0}\right)$ of $10 \mathrm{~V}$ was applied, and the real-time resistance was obtained by calculating the ratio between $V_{0}$ and $I_{0}$.

\section{Acknowledgements}

This work was financially supported by the National Natural Science Foundation of China (31870552), Hunan Provincial Technical Innovation Platform and Talent Program in Science and Technology (2018RS3092 and 2018WK4028), Outstanding Innovative Youth Training Program of Changsha (KQ1905060), Hunan Province Innovation Foundation For Postgraduate, and Science and Technology Innovation Fund for Graduate Students, from Central South University of Forestry and Technology (20181013). S.J. thanks for the funding from China Scholarship Council (CSC). Y.L. is grateful for financial support from the QMUL-SBCS startup. I.P.P. acknowledges the EPSRC M3S CDT grant (EP/L015862/1). Y.L. acknowledges the support from the Royal Society Research Grant (RGS\R1\201071).

\section{Reference}

1. H.W. Wang, J.Z. Fu, C. Wan, J.Y. Wang, A.K. Yang, C.C. Li, Q.F. Sun, Y. Cui, H.Q. Li, A binder-free high silicon content flexible anode for Li-ion batteries, Energy Environ. Sci. 13 (2020) 848-858.

2. Z. Gao, P. Liu, X.M. Fu, L.M. Xu, Y. Zuo, B. Zhang, X.M. Sun, H.S. Peng, Flexible self-powered textile formed by bridging photoactive and electrochemically active fiber electrodes, J. Mater. Chem. A, 7 (2019) 14447- 
14454.

3. Y.R. Ding, C.H. Xue, Q.Q. Fan, L.L. Zhao, Q.Q. Tian, X.J. Guo, J. Zhang, S.T. Jia, Q.F. An, Fabrication of superhydrophobic conductive film at air/water interface for flexible and wearable sensors, Chem. Eng. J. 404 (2021) 126489126496.

4. C. Wang, Y. Xiong, H.W. Wang, C.T. Jin, Q F. Sun, Naturally three-dimensional laminated porous carbon network structured short nano-chains bridging nanospheres for energy storage, J. Mater. Chem. A, 5 (2017) 15759-15770.

5. P. Wang, W.D. Wei, Z.Q. Li, W. Duan, H.L. Han, Q. Xie, A superhydrophobic fluorinated PDMS composite as a wearable strain sensor with excellent mechanical robustness and liquid impalement resistance, J. Mater. Chem. A 8 (2020) 3509-3516.

6. J. Park, Y. Lee, M.H. Barbee, S. Cho, S. Cho, R. Shanker, J. Kim, J. Myoung, M.P. Kim, C. Baig, A hierarchical nanoparticle-in-micropore architecture for enhanced mechanosensitivity and stretchability in mechanochromic electronic skins, Adv. Mater. 31 (2019) 1808148-1808157.

7. M. Kanik, S. Orguc, G. Varnavides, J. Kim, T. Benavides, D. Gonzalez, T. Akintilo, C.C. Tasan, A.P. Chandrakasan, Y. Fink, Strain-programmable fiberbased artificial muscle, Science 365 (2019) 145-150. 
8. Z. Lei, P. Wu, A highly transparent and ultra-stretchable conductor with stable conductivity during large deformation, Nat. Commun. 10 (2019) 1-9.

9. N. Matsuhisa, D. Inoue, P. Zalar, H. Jin, Y. Matsuba, A. Itoh, T. Yokota, D. Hashizume, T. Someya, Printable elastic conductors by in situ formation of silver nanoparticles from silver flakes, Nat. Mater. 16 (2017) 834-840.

10. J.C. Zhou, X.H. Guo, Z.S. Xu, Q.J. Wu, J. Chen, J. Wu ,Y.H. Dai, L. Qu, Z.X. Huang, Highly sensitive and stretchable strain sensors based on serpentineshaped composite films for flexible electronic skin applications, Compos. Sci. and Technol. 197 (2020) 108215.

11. V. Kumar, M.N. Alam, A. Manikkavel, J. Choi, D. Lee, Investigation of silicone rubber composites reinforced with carbon nanotube, nanographite, their hybrid, and applications for flexible devices, J. Vinyl Addit.Techn. (2020) 1-10.

12. H.A. Sherif, F.A. Almufadi, Fabrication and characterization of silicone rubber/multiwalled carbon nanotubes nanocomposite sensors under impact force, Sensor. Actuat. A: Phys. 297 (2019) 111479.

13. Y. Wang, K.L. Xia, H.M Wang, X.P. Liang, Z. Yin, and Y.Y. Zhang, Advanced carbon for flexible and wearable electronics, Adv. Mater. 31 (2018) 1801072.

14. W.T. Li, Y.Y Liu, Y. Bai, J. Wang, H. Pang, Anchoring ZIF-67 particles on amidoximerized polyacrylonitrile fifibers for radionuclide sequestration in wastewater and seawater, J. hazard. Mater. 395 (2020) 122692. 
15. S.S Zheng, Y. Zheng, H.G Xue, H. Pang, Ultrathin nickel terephthalate nanosheet three-dimensional aggregates with disordered layers for highly efficient overall urea electrolysis, Chem. Eng. J. 395 (2020) 125166.

16. Y. Cheng, X. Xiao, K. Pan, H. Pang, Development and application of self-healing materials in smart batteries and supercapacitors, Chem. Eng. J. 380 (2020) 122565.

17. I.S. Park, K.J. Kim, J.D Nam, W. Yim, Mechanical, dielectric, and magnetic properties of the silicone elastomer with multi-walled carbon nanotubes as a nanofiller, Polym. Eng. Sci. 47 (2007) 1396-1405.

18. J.E. Mates, I.S. Bayer, J.M. Palumbo, P.J. Carroll, C.M. Megaridis, Extremely stretchable and conductive water-repellent coatings for low-cost ultra-flexible electronics, Nat. Commun. 6 (2015) 1-8.

19. L. Wang, H. Wang, X.W. Huang, X. Song, M. Hu, L. Tang, H. Xue, J. Gao, Superhydrophobic and superelastic conductive rubber composite for wearable strain sensors with ultrahigh sensitivity and excellent anti-corrosion property, J. Mater. Chem. A 6 (2018) 24523-24533.

20. J.Y. Jeon, T.J. Ha, Waterproof electronic-bandage with tunable sensitivity for wearable strain sensors, ACS Appl. Mater. Interfaces 8 (2016) 2866-2871. 
21. A. Hassan, Y. Savaria, M. Sawan, Electronics and packaging intended for emerging harsh environment applications: A review. IEEE Trans, Very Large Scale Integr. Syst. 26 (2018) 2085-2098.

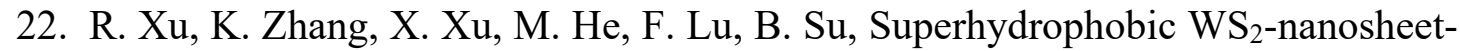
wrapped sponges for underwater detection of tiny vibration, Adv. Sci. 5 (2018) 1700655-1700664.

23. Z. Li, L. Ye, J. Shen, K. Xie, Y. Li, Strain-gauge sensoring composite films with self-restoring water-repellent properties for monitoring human movements, Compos. Commun. 7 (2018) 23-29.

24. L. Wang, J. Luo, Y. Chen, L. Lin, X. Huang, H. Xue, J.f. Gao, Fluorine-free superhydrophobic and conductive rubber composite with outstanding deicing performance for highly sensitive and stretchable strain sensors, ACS Appl. Mater. Interfaces 11 (2019) 17774-17783.

25. L. Li, Y. Bai, L. Li, S. Wang, T. Zhang, A superhydrophobic smart coating for flexible and wearable sensing electronics, Adv. Mater. 29 (2017) 17025171702524.

26. P. Wang, B. Sun, Y. Liang, H. Han, X. Fan, W. Wang, Z. Yang, A stretchable and super-robust graphene superhydrophobic composite for electromechanical sensor application, J. Mater. Chem. A 6 (2018) 10404-10410. 
27. J. Gao, L. Wu, Z. Guo, J. Li, C. Xu, H. Xue, A hierarchical carbon nanotube/ $\mathrm{SiO}_{2}$ nanoparticle network induced superhydrophobic and conductive coating for wearable strain sensors with superior sensitivity and ultra-low detection limit, J. Mater. Chem. C 7 (2019) 4199-4209.

28. J. Gao, B. Li, X. Huang, L. Wang, L. Lin, H. Wang, H. Xue, Electrically conductive and fluorine free superhydrophobic strain sensors based on $\mathrm{SiO}_{2} /$ graphene-decorated electrospun nanofibers for human motion monitoring, Chem. Eng. J. 373 (2019) 298-306.

29. X. Su, H. Li, X. Lai, Z. Chen, X. Zeng, Highly stretchable and conductive superhydrophobic coating for flexible electronics, ACS Appl. Mater. Interfaces 10 (2018) 10587-10597.

30. Q. Li, H. Liu, S. Zhang, D. Zhang, X. Liu, Y. He, L. Mi, J. Zhang, C. Liu, C. Shen, Superhydrophobic electrically conductive paper for ultrasensitive strain sensor with excellent anti-corrosion and self-cleaning property, ACS Appl. Mater. Interfaces 11 (2019) 21904-21914.

31. G. Zu, K. Kanamori, K. Nakanishi, J. Huang, Superhydrophobic ultraflexible triple-network graphene/polyorganosiloxane aerogels for a high-performance multifunctional temperature/strain/pressure sensing array, Chem. Mater. 31 (2019) 6276-6285. 
32. M. Amjadi, K.U. Kyung, I. Park, M. Sitti, Stretchable, skin-mountable, and wearable strain sensors and their potential applications: A review, Adv. Funct. Mater. 26 (2016) 1678-1698.

33. Y. Lu, S. Sathasivam, J. Song, C.R. Crick, C.J. Carmalt, I.P. Parkin, Robust selfcleaning surfaces that function when exposed to either air or oil, Science 347 (2015) 1132-1135.

34. F.A. Jamaludin, M.Z. A. Ab-Kadir, M. Izadi, N. Azis, J. Jasni, M.S. Abd-Rahman, Effects of RTV coating on the electrical performance of polymer insulator under lightning impulse voltage condition, PloS one 12 (2017) e0187892.

35. S C. Mannsfeld, B.C. Tee, R.M. Stoltenberg, C.V.H. Chen, S. Barman, B.V. Muir, A.N. Sokolov, C. Reese, Z. Bao, Highly sensitive flexible pressure sensors with microstructured rubber dielectric layers, Nat. Mater. 9 (2010) 859-864.

36. D. Zhi, Y. Lu, S. Sathasivam, I.P. Parkin, X. Zhang, Large-scale fabrication of translucent and repairable superhydrophobic spray coatings with remarkable mechanical, chemical durability and UV resistance, J. Mater. Chem. A 21 (2017) 10622-10631.

37. Z.Y. Liu, D.P. Qi, G.Y. Hu, H. Wang, Y. Jiang, G. Chen, Y.F. Luo, X.J. Loh, B. Liedberg, X.D. Chen, Surface strain redistribution on structured microfibers to enhance sensitivity of fiber-shaped stretchable strain sensors, Adv. Mater. 30 (2018) 1704229. 
38. Q. Hua, J. Sun, H. Liu, R. Bao, R. Yu, J. Zhai, C. Pan, Z.L. Wang, Skin-inspired highly stretchable and conformable matrix networks for multifunctional sensing, Nat. Commun. 9 (2018) 1-11.

39. Q. Liu, J. Chen, Y. Li, G. Shi, High-performance strain sensors with fish-scalelike graphene-sensing layers for full-range detection of human motions, ACS nano 10 (2016) 7901-7906.

40. X. Liao, Q. Liao, Z. Zhang, X. Yan, Q. Liang, Q. Wang, M. Li, Y. Zhang, A highly stretchable $\mathrm{ZnO} @$ fiber-based multifunctional nanosensor for strain/temperature/UV detection, Adv. Funct. Mater. 26 (2016) 3074-3081.

41. G. Shi, Z. Zhao, J.H. Pai, I. Lee, L. Zhang, C. Stevenson, K. Ishara, R. Zhang, H. Zhu, J. Ma, Highly sensitive, wearable, durable strain sensors and stretchable conductors using graphene/silicon rubber composites, Adv. Funct. Mater. 26 (2016) 7614-7625.

42. J. Li, S. Zhao, X. Zeng, W. Huang, Z. Gong, G. Zhang, R. Sun, C.P. Wong, Highly stretchable and sensitive strain sensor based on facilely prepared threedimensional graphene foam composite, ACS Appl. Mater. Interfaces 8 (2016) 18954-18961.

43. Q. Guo, Y. Luo, J. Liu, X. Zhang, C. Lu, A well-organized graphene nanostructure for versatile strain-sensing application constructed by a covalently bonded graphene/rubber interface, J. Mater. Chem. C 6 (2018) 2139-2147. 
44. H. Yang, X. Yao, Z. Zheng, L. Gong, L. Yuan, Y. Yuan, Y. Liu, Highly sensitive and stretchable graphene-silicone rubber composites for strain sensing, Compos. Sci. Technol. 167 (2018) 371-378.

45. Y. Li, B. Zhou, G. Zheng, X. Liu, T. Li, C. Yan, C. Cheng, K. Dai, C. Liu, C. Shen, Continuously prepared highly conductive and stretchable SWNT/MWNT synergistically composited electrospun thermoplastic polyurethane yarns for wearable sensing, J. Mater. Chem. C 6 (2018) 2258-2269.

46. T. Wang, Y. Zhang, Q. Liu, W. Cheng, X. Wang, L. Pan, B. Xu, H. Xu, A selfhealable, highly stretchable, and solution processable conductive polymer composite for ultrasensitive strain and pressure sensing, Adv. Funct. Mater. 28 (2018) 1705551-1705562.

47. S. Wang, K. Chen, M. Wang, H.Li, G. Chen, J. Liu, L. Xu, Y. Jian, C. Meng, X. Zheng, Controllable synthesis of nickel nanowires and its application in high sensitivity, stretchable strain sensor for body motion sensing, J. Mater. Chem. C 6 (2018) 4737-4745.

48. L. Lu, Y. Zhou, J. Pan, T. Chen, Y. Hu, G. Zheng, K. Dai, C. Liu, C. Shen, X. Sun, Design of helically double-leveled gaps for stretchable fiber strain sensor with ultralow detection limit, broad sensing range, and high repeatability, ACS Appl. Mater. Interfaces 11 (2019) 4345-4352. 
49. J. Huang, J. Zhou, Y.M. Luo, G. Yan, Y. Liu, Y.P. Shen, Y. Xu, H.L. Li, L.B. Yan, G.H. Zhang, Y.Q. Fu, H.G. Duan, Wrinkle-enabled highly stretchable strain sensors for wide-range health monitoring with a big data cloud platform, ACS Appl. Mater. \& Interfaces 12 (2020) 43009-43017.

50. M.C. Qu, Y.J. Qin, Y. Sun, H.G. Xu, D.W. Schubert, K. Zheng, W. Xu, F. Nilsson, Biocompatible, flexible strain sensor fabricated with polydopaminecoated nanocomposites of nitrile rubber and carbon black, ACS Appl. Mater. \& Interfaces 12 (2020) 42140-42152.

51. H.J. Zhang, W.Q. Han, K. Xu, Y. Zhang, Y.F. Lu, Z.T. Nie, Y.H. Du, J.X. Zhu, W. Huang, Metallic sandwiched-aerogel hybrids enabling flexible and stretchable intelligent sensor, Nano Lett. 20 (2020) 3449-3458.

52. Y. Wang, Y. Jia, Y. Zhou, Y. Wang, G. Zheng, K. Dai, C. Liu, C. Shen, Ultrastretchable, sensitive and durable strain sensors based on polydopamine encapsulated carbon nanotubes/elastic bands, J. Mater. Chem. C 6 (2018) 81608170.

53. L. Wang, Y. Chen, L. Lin, H. Wang, X. Huang, H. Xue, J. Gao, Highly stretchable, anti-corrosive and wearable strain sensors based on the PDMS/CNTs decorated elastomer nanofiber composite, Chem. Eng. J. 362 (2019) 89-98.

54. S.S. Jia, X.H. Lu, S. Luo, Y. Qing, N. Yan, Y.Q. Wu, Efficiently texturing hierarchical epoxy layer for smart superhydrophobic surfaces with excellent 
durability and exceptional stability exposed to fire, Chem. Eng. J. (2018). 348 (2018) 212-223.

55. W. Zhang, T. Xiang, F. Liu, M. Zhang, W. Gan, X. Zhai, X. Di, Y. Wang, G. Liu, C. Wang, Facile design and fabrication of superwetting surfaces with excellent wear-resistance, ACS Appl. Mater. Interfaces 9 (2017) 15776-15784.

56. X. Zhang, D. Zhi, L. Sun, Y. Zhao, M.K. Tiwari, C.J. Carmalt, I.P. Parkin, Y. Lu, Super-durable, non-fluorinated superhydrophobic free-standing items, J. Mater. Chem. A 6 (2018) 357-362.

57. D. Richard, D. Quéré, Bouncing water drops, Europhys. Lett. 50 (2000) 769-775. 